\title{
An effective way to eradicate Ebola
}

\author{
Sicheng $\mathrm{Zhu}^{1, \mathrm{a}}$ \\ ${ }^{1}$ School of North China Electric Power University, Hebei 071000, China; \\ assc4572@126.com
}

Keywords: delivery, manufacture, Ebola, distribution

\begin{abstract}
In this papers, we make a study on the delivery, manufacture of drugs and vaccines to treat Ebola virus as well as the impact on Ebola epidemic brought from drugs and vaccines. Firstly, we build a model to estimate the demand of drugs and vaccines at the next delivery time after considering the capacity of producing drugs and vaccines, thus we make plans of manufacture. At the same time, taking the contact between the neighboring countries and three hard-hit countries into account, we discuss about the probability of disease prevalence among its neighboring countries. In view of the existing number of patients, we get further developments of the epidemic. Secondly, it is universally acknowledged that drugs can suppress the spread of the virus directly, vaccines can decline a person's morbidity rate on the whole. We amend the morbidity rate and use the correction to calculate the number of people to be infected possibly in the future. Then we forecast the continuous changed trend of what the number of infected people will be in the infected areas.
\end{abstract}

\section{Introduction}

The Ebola virus is a kind of potent virus that caused Ebola Hemorrhagic fever (EBHF) in humans and primates [1], having a very high mortality. Since Month 2015, an ongoing outbreak of Ebola in West Africa has infected at least 22057 people in Guinea, Sierra Leone, and Liberia, including 8795 who died [6]. We know that the world medical association has announced that their new medication could stop Ebola and cure patients whose disease is not advanced. The problem we are faced with is how to build a realistic, reasonable, and useful model to eradicate the Ebola virus.

In order to eradicate the Ebola virus, we need to consider a series of factors, which mainly contain: the quantity of people infected, the possibility of normal people being infected, the interaction between main infected areas, the therapeutic effect of drugs use, the effect of the medical conditions in infected areas,the capacity of vaccine and drug,the conditions of transportation [4].

\section{Models}

After thinking the actual situation, we realize that it requires to transport quantities of drugs and vaccines multiple times and the transport has a certain cycle. To coincide with the treatment work, we need to make the manufacture plan for drugs and vaccines, which requires us to expect the demand for each cycle of transport. According to the above two models, we can know the speed of disease transmission under the premise of not considering the effects of drugs. In order to get the right anticipated demand, we need to take the effects of drugs and vaccines into account.

\section{Establish model}

When the drugs are sufficient, it can meet the requirement of treatment for any patient who can be cured.When lack of drugs, we should take the priority into account, which asks for us ought to transport the drugs to intensive infected areas. Then according to the areas' probability of people being infected, we can calculate the demand for drugs. Finally we distribute the drugs in view of principles on-demand [2].

First, we get every country's population density $\eta_{i}$ (unit: population $/ \mathrm{km}^{2}$ ). Then combine with various culture, religious beliefs, social environment and natural environment among different countries to determine a correction factor $k_{1}$, because different countries may differ as a result of these factors and thus indirectly affect the people's daily communication habits [3]. 
When not considering the effects of drugs and vaccines, we can define a person's morbidity rate in country $i$ as:

Where

$$
P_{i}=\eta_{i} \cdot k_{1} \cdot \frac{N_{i-i l l}}{N_{i-\text { total }}} \cdot k_{2}
$$

$N_{i-i l l}$ stands for the number of infected people in country $i$

$N_{i-t o t a l}$ stands for the total population in country $i$

$k_{2}$ stands for a comprehensive coefficient, combined with the sanitation, medical condition, national policy and state of the economy.

When consider the effects of drug, On the one hand, the use of drugs would inhibit the spread of the virus, leading to the lower transmission of the virus, that is lower the comprehensive coefficient $k_{2}$ [5]. We consider the rates of drug use $\alpha$ and get a fixed comprehensive coefficient to replace $k_{2}$ :

$$
k_{N}=k_{2} \cdot(1-\alpha) \cdot \lambda
$$

Where $\lambda$ stands for the ability of drugs to inhibit the spread of the virus.

On the other hand, the use of drugs will cure some patients, so we should exclude these people from the projected infections [7].

When consider the effect of vaccine, The use of vaccines will reduce the morbidity rate on the whole, so we should amend the morbidity rate $P_{i}$. Because the vaccines cannot come into force immediately and it will take some time. We define the valid time range $\left[t_{1}, t_{2}\right]$. When not in this time range, it does not need revisions. When during this time range, we need to make the following amendments:

$$
P_{i}^{\prime}=\eta_{i} \cdot k_{1} \cdot \frac{N_{i-i l l}}{N_{i-\text { total }}} \cdot k_{N} \cdot\left(1-\beta_{i} \cdot \frac{t-t_{1}}{t_{2}-t_{1}}\right)
$$

Where $\beta_{i}$ stands for the proportion of people receiving vaccination in country $i$ Therefore, we can get the following equations:

$$
\left\{\begin{array}{cc}
k_{N}=k_{2} \cdot(1-\alpha) \cdot \lambda & \left(0<t<t_{1}\right) \\
P_{i}^{\prime} \cdot k_{1} \cdot \frac{N_{i-i l l}}{N_{i-\text { total }}} \cdot k_{N} & \left(t_{1}<t<t_{2}\right) \\
\eta_{i} \cdot k_{1} \cdot \frac{N_{i-i l l}}{N_{i-\text { total }}} \cdot k_{N} \cdot\left(1-\beta_{i} \cdot \frac{t-t_{1}}{t_{2}-t_{1}}\right) & \\
N_{T-i-\text { ill }}=N_{0-i-i l l}+N_{i-\text { total }} \cdot \int_{0}^{T} P_{i}^{\prime} d t-N_{i-\text { total }} \cdot \alpha-N_{i-\text { death }}
\end{array}\right.
$$

Where $T$ stands for the time required for vaccines to take effect, that is $T=t_{2}-t_{1}$.

To solve the model, we require $P_{i}^{\prime} \leq e$, where $e$ is a extremely small constant.

By solving the equations above, we can calculate the demand for drugs and vaccines.

Further more, when the number of vaccines are sufficient, in addition, we consider the number of vaccines given to the areas around the infected country.

$$
A_{j}=\sum_{i=1}^{n} R_{i j} \cdot \frac{N_{j-i l l}}{N_{j-\text { total }}} \cdot k_{3}
$$

Where $k_{3}$ is combined with exchange among regions and geographical features. 


\section{The result}

According to practical condition of the illness, we choose three main affected countries, followed by Guinea, Liberia and Sierra Leone.

- The drugs

By solving the model, we can get the demand for drugs and the proportion of demand in these three countries.

Tab 1: the demand of drugs and the proportion in three countries

\begin{tabular}{llll}
\hline & Guinea & Liberia & Sierra Leone \\
\hline The demand & 1031 & 4999 & 7464 \\
Proportion & $7.64 \%$ & $37.05 \%$ & $55.31 \%$ \\
\hline
\end{tabular}

Form the table, we can see that the illness is most serious in Sierra Leone, the corresponding demand for drugs is also the biggest.

When drugs are inadequate, it should be distributed according to the proportion. When drugs are sufficient, it should be distributed according to the actual demand.

- The vaccines

We search the country's population and land area from the internet, getting the population density. In order to facilitate the processing, we take $k_{1}=0.3$ to get the average number of people of a person contact per day

In order to ensure the morbidity we make a linear fitting with the morbidity and the average daily number of people contact. We take Guinea for example:

The general model is : $f(x)=k_{2} \cdot x$

Coefficients (with 95\% confidence bounds): $k_{2}=0.001582 \quad(0.0013,0.001864)$

Using the same method, we can get the other two countries' linear fitting results. Combined with average daily number of patients of a people contact, we can calculate the probability of being infected:

Tab 2: the morbidity rate

\begin{tabular}{cccc}
\hline & Guinea & Liberia & Sierra Leone \\
\hline $\begin{array}{c}\text { Average daily number of } \\
\text { patients of a people contact }\end{array}$ & 0.00125653 & 0.01238124 & 0.02914631 \\
Coefficient $k_{2}$ & 0.00158200 & 0.00114000 & 0.00054390 \\
Morbidity rate & 0.00000199 & 0.00001411 & 0.00001585 \\
\hline
\end{tabular}

In order to determine the percentage of people who receive the vaccines, we set the constant $e=0.000001$, thus we can obtain a critical value of the proportion. Then multiplied by total population of the country (we take an integer) we get the demand for vaccines.

Tab 3: the proportion of vaccination and the demand for vaccines

\begin{tabular}{lccc}
\hline & Guinea & Liberia & Sierra Leone \\
\hline The proportion of vaccination & 0.49694 & 0.92915 & 0.93692 \\
The demand for vaccines & $5,565,716$ & $3,995,351$ & $5,715,208$ \\
The proportion of demand for vaccines & 0.36434 & 0.26154 & 0.37412 \\
\hline
\end{tabular}

When the vaccines are sufficient,we give vaccines to the areas around the infected country.

From the geography of West Africa from Google Map, we can get the eight countries adjacent to the three infected countries, namely Nigeria, Senegal, Mali, Gambia, Guinea Bissau, Ivory Coast, Benin and Niger.We can get the matrix $R_{i j}$ from the map directly:

$$
R_{i j}=\left(\begin{array}{cccccccc}
0 & 1 & 1 & 0 & 1 & 1 & 0 & 0 \\
0 & 0 & 0 & 0 & 0 & 1 & 0 & 0 \\
0 & 0 & 0 & 0 & 0 & 0 & 0 & 0
\end{array}\right)
$$


To solve the equations, for example, we take $\lambda=0.8, \alpha=0.5, \beta=0.5 T=7, t_{1}=3, t_{2}=10$. The corresponding results are shown in the following table.

Tab 4: the demand for drugs and vaccines

\begin{tabular}{lccc}
\hline & Guinea & Liberia & Sierra Leone \\
\hline The demand for drugs & 523 & 2551 & 3858 \\
The demand for vaccines & 89889 & 3702836 & 5355541 \\
\hline
\end{tabular}

Combined with the actual situation, to simplify the processing, we take $k_{3}=0.37$. Finally we get a series of corresponding results about the eight countries.

Based on the results of model above, we get the corresponding plan of transport and production. For the constraints of capacity, we cannot produce vaccines and drugs as expected. We conclude the changes of the development of epidemic according to the actual production. We take the trend of Guinea for example to reflect the changes of the spread of the epidemic in the three countries.

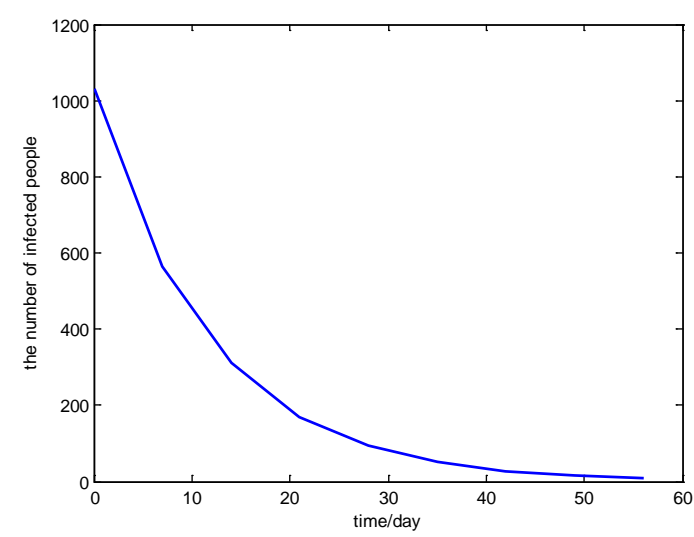

Fig 1: the change of the severity of epidemic in Guinea

The other two curves are in the same trend. From these curves, with the multiple launch of drugs and vaccines, we can see that the epidemic is going to be controlled gradually. At the time of 60 days, the number of infected people are almost zero, which demonstrates that vaccines and drugs have taken effect, that means the Ebola virus have been eradicated. It is basically in line with our expected results, which shows that our approach is feasible.

\section{Conclusion: The manufacture plans}

The plans of manufacture are based on the predicted demand for drugs and vaccines. Due to the patients are concentrated at the hospitals, the drugs should be transported to hospitals in major cities and specific distribution should be assigned according to patients' actual severity. Vaccines should be mainly put into densely populated cities, and the areas bordered on the infected countries.

After taking the effects of drugs and vaccines into account, we are able to get a reasonable demand for drugs and make an appropriate manufacture plan of drugs and vaccines. In the model three, we randomly distribute vaccines and drugs, however, in fact, the government will distribute drugs and vaccines according to the actual condition of the country.

The results show that Sierra Leone needs 3,858 drugs and 5355541 vaccines, which is in line with the actual situation because the the severity of the Ebola is most serious in Sierra Leone. In contrast, Guinea needs 523 drugs, 89,889 vaccines for the severity of the Ebola is the lightest.

\section{References}

[1] Li Chao, Yang Ming, Mou di, Sui Haitian, Zhao Jian,Meng Yujie, Tu wenxiao. Analysis of characteristics of Ebola virus epidemic from 2013 to 2014 in West Africa [J] Disease Surveiliance, 2014, 29(11): 925-928

[2] Yu Lei, Xue Huifeng, Li Gang. Research of Epidemic Spread Model [J] Computer Simulation, 
2007, 24(04): 57-60

[3] Zhang Fa, Li Lu, Xuan Huiyu. Survey of transmission models of infectious diseases [J] Systems Engineering-Theory\&Practice, 2011, 31(9): 1736-1744

[4] http://blog.sciencenet.cn/blog-528739-865966.html (7 Feb,2015)

[5] http://www.chinacdc.cn/rdwd/201408/t20140809_101029.htm (7 Fed, 2015)

[6] http://en.wikipedia.org/wiki/Ebola_virus_epidemic_in_West_Africa(7 Feb, 2015)

[7] http://www.wma.net/en/30publications/10policies/e21/index.html (7 Feb,2015) 